\title{
From Discrete 1 to 10 Towards Continuous 0 to 10: The Continuum Approach to Estimating the Distribution of Happiness in a Nation
}

\author{
Wim Kalmijn
}

Accepted: 25 September 2011/Published online: 13 October 2011

(C) The Author(s) 2011. This article is published with open access at Springerlink.com

\begin{abstract}
Happiness is often measured in surveys using responses to a single question with a limited number of response options, such as 'very happy', 'fairly happy' and 'not too happy'. There is much variety in the wording and number of response options used, which limits comparability across surveys. To solve this problem, descriptive statistics of the discrete distribution in the sample are often transformed to a common discrete secondary scale, mostly ranging from 0 to 10 . In an earlier publication we proposed a method for estimating statistics of the corresponding continuous distribution in the population (Kalmijn 2010). In the present paper we extend this method to questions using numerical response scales. The application of this 'continuum approach' to results obtained using the often used 1-10 numerical scale can make these comparable to those obtained on the basis of verbal response scales.
\end{abstract}

Keywords Happiness - Life satisfaction - Measurement - Statistics · Numerical scale · Continuum approach

\section{Measurement of Happiness}

Happiness, or any other aspect of quality of life, in a nation or some other collectivity is generally measured at the individual level. Usually, it is done by drawing a sample from the population under study and by asking each respondent in the sample to answer a single closed question with a limited number of response options. These options are either "verbal" (i.e. textual), numerical (using integer code and/or rank order numbers) or pictorial (e.g. using "smileys"); their number per question varies between 2 and 12 in far most cases.

The most frequently chosen method to measure happiness in a nation is by self-report of the respondents to a single question, e.g. "Taking all together, how happy are you with your life these days?". The respondent has to select one out of a number of response

W. Kalmijn $(\bowtie)$

Erasmus University Rotterdam, Rotterdam, The Netherlands

e-mail: kalmijn@fsw.eur.nl 
alternatives. The World Database of Happiness (Veenhoven 2011), further abbreviated WDH, includes a collection of combinations of a leading question and the corresponding set of the various alternatives presented to the respondents in happiness studies; these combinations are referred to in the WDH as "measures of happiness".

An increasing number of surveys applies ten-step, or ten-point, "numerical scales". In these surveys, the respondent has to select one of the integer numbers, presented e.g. as follows:

\begin{tabular}{rrrrrrrrrrr}
\hline $\begin{array}{r}1 \\
\text { very unhappy }\end{array}$ & 3 & 4 & 5 & 6 & 7 & 8 & 9 & $\begin{array}{r}10 \\
\text { very happy }\end{array}$ \\
\hline
\end{tabular}

The average of the ten ratings, each weighted with its relative frequency as its weight, is the sample average value, which in turn is used to compute the standard deviation within the sample in the usual way. These two statistics are considered to characterize the happiness distribution as it is measured in the sample. In all these cases, the measurement procedure results in a frequency distribution of a discrete response variable in the sample. There is an almost complete consensus among happiness investigators on the appropriateness of this approach.

\section{Happiness Distribution in Samples and Populations}

In the past however, researchers in this field paid very little attention to the conversion of this sample distribution data into information about the corresponding happiness distribution in the population represented by this sample, at least explicitly. We have not found any investigation into this surprising phenomenon, so our possible explanations of this are necessarily speculative.

The fixation that social scientists have on hypothesis testing may prevent them seeing parameter estimation to be an at least equally valuable component of statistical inference. Another possible explanation may be found in the fact that most initial studies in this field are correlational studies based on primary data, i.e. data collected for this specific study within the same sample of individuals. If a statistically significant correlation is established within this sample, it is simply declared to apply to the population as well. The application of "secondary" data at a later stage, i.e. data collected from different samples for a different or for a general purpose, has not given rise to a different approach.

Three methods for the conversion of sample data into information on the happiness distribution in the population are in use:

(1) simple generalization

(2) linear or non-linear transformation to a scale from 0 to 10

(3) application of the continuum approach.

In this paper we describe these methods and give rules for the conversion of the outcomes of results according of one of these methods.

\section{Simple Generalization}

In this approach, the values of statistics as these have been obtained from the sample are declared to apply also to the population as a whole. Sometimes, $95 \%$ confidence limits for 
the true but unknown mean happiness value of the population are reported; these are calculated as $m \pm 2 s / \sqrt{ } N$, where $m=$ sample average value, $s=$ sample standard deviation and $N=$ sample size.

\section{Linear Transformation to a Common Secondary $[0,10]$ Scale}

Each measure of happiness has its own number of response alternatives. In most measures this number is within the range of 3 to 11 . This was not seen as a problem until data had to be compared that had been obtained using different scales of happiness measurement, in particular those with different numbers of response options. To make the results obtained from different measures comparable, Veenhoven (1993:115) proposed to transform the various primary verbal scales into one common secondary scale, for which a scale of $0-10$ has become the usual choice. The most unhappy (happy) rating is given the secondary value " 0 " ("10") and the positions of the other ratings on the secondary scale result from the proportionally stretching the scale to the new range $[0,10]$. This procedure can be described as "direct stretch(ing)" or as rescaling on the basis of linear transformation of the ratings of the scale to cover the common $[0,10]$ interval.

It implies that in the case of a 10-point primary scale $1 \rightarrow 0$ and $10 \rightarrow 10$. This transformation can be considered to be a two-step process: a shift $[1,10] \rightarrow[0,9]$, followed by a stretching $[0,9] \rightarrow[0,10]$. The consequences are that the statistics $m$ and $s$ as defined above are transformed as follows:

$$
\begin{aligned}
m \rightarrow m_{2} & =(10 / 9)(m-1), \text { and } \\
s \rightarrow s_{2} & =(10 / 9) s .
\end{aligned}
$$

Note that this secondary scale is still a 10-point scale, but that the distance between consecutive ratings has been increased from 1 to 10/9. This linear transformation is applied in many studies included in the WDH.

A similar approach is proposed by others, who transform the ratings and average values into the corresponding percentage of the maximum possible score of the measurement scale. Examples include Cummins (1995) and Mazaheri and Theuns (2009). If the minimum score on their scale(s) is assumed to be zero, these statistics and those according to Veenhoven are identical, but for a factor 10 .

\section{Nonlinear Rescaling Methods}

The linear transformation method has several weak points, in particular in the case of a verbal primary rating scale with only a few ratings. The two most fundamental objections are that (1) the text of the response options is fully ignored in this way, and (2) the linear transformation maintains equidistance between consecutive ratings without any justification. Therefore, Veenhoven c.s. $(1993 ; 108-114)$ introduced "Thurstone ratings" as an alternative rescaling method. In this method, a response option like "pretty happy" is given a 'universal secondary rating', in this particular case 6.7 on a $[0 ; 10]$ continuum. This solution, however, was also felt to be unsatisfactory.

An alternative approach is developed in the International Happiness Scale Interval Study (Veenhoven 2009), in which a response option, e.g. "pretty happy" is assumed to cover a subset of contiguous happiness values, one subset for each response option. 
If happiness is measured using a four-step verbal scale, the continuum $[0,10]$ of the happiness variable is partitioned into four contiguous subintervals, each of which corresponds to one of the response options of the measured happiness. A common series of verbal response options is \{ "very happy", "pretty happy", "not too happy", "very unhappy" $\}$. These four options do not neatly fit quartels on the $[0,10]$ continuum, hence a first step is to estimate where the boundaries are.

The leading question with the set of alternative response options is presented to a group of native speakers, who were asked to identify the boundary between successive response options, e.g. between "pretty happy" and "not too happy" on a $[0,10]$ continuum, in which "0" ("10") represents the least (most) happy situation they could imagine and ignoring their own happiness situation. Each of these "judges" estimates the three boundaries on the $[0,10]$ interval; they do so in the context of a particular series of response options in a particular language. The opinions of all judges on the same boundary are averaged, resulting in e.g. the happiness value 6.3 as the dividing point between "not too happy" and "pretty happy". The mid-interval value of each sub-interval is adopted as the secondary rating of this particular response option within the context of this particular leading question and this particular set of response alternatives, all formulated in this particular language and in this particular period of time.

\section{Happiness as a Continuous Latent Variable}

None of the above approaches allows a link to be established between the distribution of the happiness variable as measured in the sample and the distribution of the happiness variable in the population.

All methods mentioned above implicitly consider happiness in the population as a discretely distributed variable, but in the continuum approach of Kalmijn (2010; Ch. 6) the existence of a latent happiness variable is postulated, which is assumed to be continuous with the interval $[0,10]$ as its domain. The boundary value " 0 " ("10") corresponds to the least (most) happy situation the respondent can conceive for him/herself. Happiness information on a population gained using this approach is always information on the distribution of this latent variable. This continuum approach uses the sub-interval boundary estimates of the various response options as they are reported by the judges in the Happiness Scale Interval Study. The average values of each boundary form the basis for the conversion procedure as mentioned above.

The methodological aspects of this Scale Interval Study and the continuum approach are discussed in e.g. Kalmijn et al. (2011).

Happiness described in this way is the variable in which quality-of-life investigators are interested and its parameters or other characteristics are (to be) applied in correlational studies of happiness. The continuum approach provides the estimates of these characteristics on the $[0,10]$ continuum. More precisely: it is a method to convert the sample observations of happiness, as it is measured by using a discrete ordinal scale of measurement, into estimates of the parameters of the happiness distribution in the population represented by this sample.

The justification of this approach is the underlying assumption that, when happiness is measured using different scales in different languages, the results can be converted into estimated characteristics of population distributions that are equal, at least approximately, in this way providing a more valid basis for correlational happiness studies. 


\section{The Conversion Procedure in the Continuum Approach}

The conversion of the sample measurement data into the estimated characteristics of the population happiness distribution is the result of four different contributions:

\begin{tabular}{|c|c|c|}
\hline & Contribution & Contributor(s) \\
\hline 1. & Choice of "happiness measure" (leading question + response options) & $\begin{array}{l}\text { Survey } \\
\text { conductor }\end{array}$ \\
\hline 2. & Individual happiness frequency distribution (application phase) & Respondents \\
\hline 3. & Estimation of boundary positions (construction or calibration phase) & Judges \\
\hline 4. & Choice of the distribution model of the latent population happiness variable & Methodologis \\
\hline
\end{tabular}

Two alternative models have been developed for making the choice in the fourth contribution: a semi-continuous model and a fully continuous one.

The domain of the latent happiness variable, usually $[0,10]$, in the semi-continuous model is partitioned into $k$ intervals, $k$ being the number of response options. A uniform distribution of the latent happiness variable is postulated to exist between each pair of consecutive interval boundaries. The probability density function is not continuous, but it is a step function with a step at each boundary value of the latent variable.

Until now, the continuum approach has been described only as an application to verbal scales. In the present paper we extend this approach with the application to a discrete numerical $\{1,2, \ldots, 10\}$ scale. The third contribution in the above listing is not made by judges, but by the methodologist. An obvious choice is the partitioning of the $[0,10]$ continuum into ten intervals with equal width. In the latter case, consecutive ratings are assumed to be equidistant, which looks admissible, in particular when these scales are presented pictorially that includes this equidistance. The first interval is $[0,1]$ and the seventh is $(6,7]$. It is assumed that each respondent who judges his/her happiness situation to be represented by a value somewhere in the seventh interval will report a rating " 7 ". The exact position of the respondent's happiness within this interval, however, is unknown. All we can do, and should do, is to make assumptions on the distribution of the latent happiness variable over each of the ten intervals. We follow the proposal of Kalmijn (2010:177) that a uniform distribution of the happiness should be assumed within each of the ten intervals, each with its own density (Fig. 1).

In this way each happiness value is mapped onto one of the ten ratings of the primary sale of measurement and each rating is treated as the mid-value of the corresponding interval. So the relative frequency of the rating " 7 " is an estimate of its relative share of the happiness distribution in the population.

An unbiased estimator of the mean value of this happiness distribution is obtained under these assumptions as $m-0.5$. A formal proof is given in the "Appendix", but this result is understandable if one assumes that each respondent is inclined to choose the rating that is closest to his/her happiness experience. In this way a rating of " 4 " is chosen by all respondents with a perceived happiness value between 3.5 and 4.5 . The rating " 1 " belongs to the interval $0.5-1.5$ and " 10 " to $9.5-10.5$. The actual scale is then a $[0.5,10.5]$ scale, which requires a shift over a distance of 0.5 to obtain a variable that is distributed on the $[0$, 10] interval.

In the "Appendix" we also prove that $s^{2}+1 / 12$ is an unbiased estimator of the population variance in the model as chosen above. 


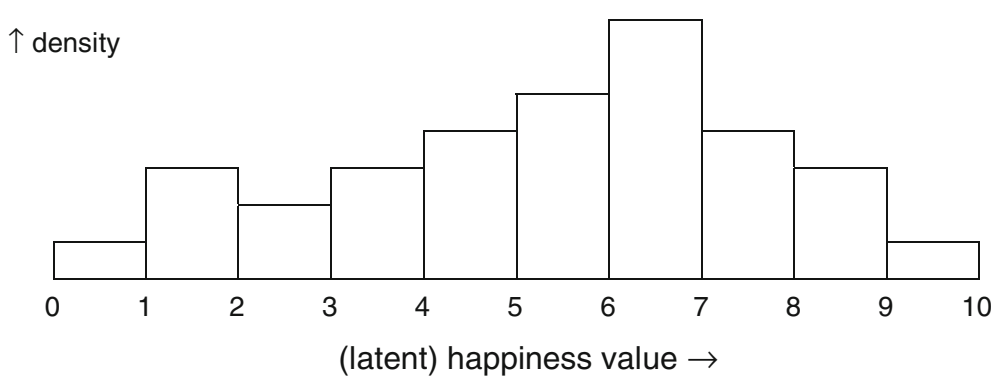

Fig. 1 Semi-continuous distribution of a continuous latent happiness variable on the basis of measurement using a discrete 1-10 ten-point scale

Since we consider happiness to be a continuous variable rather than a discrete one, we prefer the continuum approach, it being the more valid one to use in this situation. As a consequence, we recommend statistics obtained by direct stretching $[1,10] \rightarrow[0,10]$ to be replaced with $m-0.5$ and $s^{2}+1 / 12$ respectively, where $m$ and $s$ are the sample average value and the standard deviation on the primary $[1,10]$ scale.

As is apparent, this conversions require a knowledge of the 'primary' sample mean and variance (or standard deviation) only and not a knowledge of the detailed original happiness frequency distribution within the sample. This is an advantage over the alternative model, the fully continuous model on the basis of a beta distribution of the latent happiness variable. Application of the latter model requires the knowledge of the complete sample distribution; knowledge of the average value and the standard deviation of the sample only is not sufficient. The calculation of the population distribution parameter estimates is more complicated; see Kalmijn (2010; 131-133 and 160-162) for a description of this calculation.

\section{Conclusion}

The happiness distribution in a population can be described in terms of the parameters of the distribution of a continuous random variable on the $[0,10]$ interval. If happiness is measured in a sample using a discrete 10-point scale, the parameters, the mean value and the variance, of the population happiness distribution can be estimated from the corresponding estimated parameters of the sample distribution $m$ and $s^{2}$, both calculated in the conventional way.

The unbiased estimated population mean happiness value equals $m-0.5$ and the unbiased estimated population variance $=s^{2}+1 / 12$.

Open Access This article is distributed under the terms of the Creative Commons Attribution Noncommercial License which permits any noncommercial use, distribution, and reproduction in any medium, provided the original author(s) and source are credited.

\section{Appendix: Estimating the Model Parameters of the Semi-Continuous Latent Variable}

The model is based on a latent happiness $H$ as a real-valued continuous random variable on the closed interval $[0,10] \subset \mathbb{R}$. 
The domain of $H$ is partitioned into 10 intervals of equal width $(=1)$. Within each of these intervals $H$ is distributed uniformly, so the density function of $H$ is

$$
\begin{gathered}
g(h)=\pi_{j} \text { for } j-1<H \leq j \quad j=1(1) 10 \text { with } \forall j \quad 0 \leq \pi_{j} \leq 1 \text { and } \\
\qquad \sum_{j=1}^{10} \pi_{j}=1
\end{gathered}
$$

This distribution is called "semi-continuous", because the random variable $H$ is continuous, but its density is not continuous for the values $H=1(1) 9$.

The expected value of this variable $H$, denoted $\mu$, is:

$$
\mu:=\boldsymbol{E}\{H\}:=\int_{0}^{10} g(h) h \mathrm{~d} h=\sum_{j=1}^{10} \int_{j-1}^{j} \pi_{j} h \mathrm{~d} h=\sum_{j=1}^{10} \pi_{j}\left(\frac{1}{2} j^{2}-\frac{1}{2} j^{2}+j-\frac{1}{2}\right),
$$

or

$$
\mu=\sum_{1}^{10} j \pi_{j}-\frac{1}{2} \Leftrightarrow \sum_{1}^{10} j \pi_{j}=\mu+\frac{1}{2}
$$

The variable $H$ has a variance:

$$
\sigma^{2}:=\boldsymbol{E}\left\{(H-\mu)^{2}\right\}=\boldsymbol{E}\left\{H^{2}\right\}-\mu^{2}
$$

From:

$$
\boldsymbol{E}\left\{H^{2}\right\}:=\int_{0}^{10} g(h) h^{2} \mathrm{~d} h=\sum_{1}^{10} \int_{j-1}^{j} \pi_{j} h^{2} \mathrm{~d} h=\sum_{1}^{10} \pi_{j}\left(\frac{1}{3} j^{3}-\frac{1}{3} j^{3}+j^{2}-j+\frac{1}{3}\right)
$$

or, in combination with (4):

$$
\boldsymbol{E}\left\{H^{2}\right\}=\sum_{1}^{10} j^{2} \pi_{j}-\sum_{1}^{10} j \pi_{j}+\sum_{1}^{10} \frac{1}{3} \pi_{j}=\sum_{1}^{10} j^{2} \pi_{j}-\left(\mu+\frac{1}{2}\right)+\frac{1}{3}=\sum_{j} j^{2} \pi_{j}-\mu-\frac{1}{6},
$$

it follows that:

$$
\sigma^{2}=\sum_{1}^{10} j^{2} \pi_{j}-\mu^{2}-\mu-\frac{1}{6} \Leftrightarrow \sum_{1}^{10} j^{2} \pi_{j}=\sigma^{2}+\mu^{2}+\mu+\frac{1}{6} .
$$

Now we focus to the sample. For typographic convenience, we will omit the index "1" in the statistics $m_{1}, s_{1}$ and $s_{1}^{2}$.

The ten counted relative frequencies $\left\{f_{j} \mid j=1(1) 10\right\}$ should be considered to be unbiased estimators $\hat{\pi}_{j}$ of the corresponding $\left\{\pi_{j}\right\}$, so

$$
\boldsymbol{E} f_{j}=\boldsymbol{E} \hat{\pi}_{j}=\pi_{j} \quad j=1(1) 10 .
$$

The average sample happiness value: 


$$
m:=\sum_{1}^{10} j f_{j}
$$

as an estimator of $\mu$ is positively biased in the above model in view of (4):

$$
\boldsymbol{E} m=\sum_{j} j \boldsymbol{E} f_{j}=\sum_{j} j \pi_{j}=\mu+\frac{1}{2},
$$

but this bias can be removed easily by replacing $m$ with $m-1 / 2$.

The sample variance $s^{2}$ is calculated as:

$$
s^{2}=\frac{N}{N-1} \sum_{j=1}^{10} f_{j}(j-m)^{2}
$$

So

$$
\frac{N-1}{N} s^{2}:=\sum_{j} f_{j}(j-m)^{2}=\sum_{j} j^{2} f_{j}-2 m \sum_{j} j f_{j}+m^{2} \sum_{j} f_{j}=\sum_{j} j^{2} f_{j}-m^{2} .
$$

Taking expectations and combining with (7) gives:

$$
\begin{aligned}
& \frac{N-1}{N} \boldsymbol{E} s^{2}=\sum_{j} j^{2} \boldsymbol{E} f_{j}-\boldsymbol{E} m^{2}=\sum_{j} j^{2} \pi_{j}-\boldsymbol{E} m^{2}=\sigma^{2}+\mu^{2}+\mu+\frac{1}{6}-\boldsymbol{E} m^{2} . \\
& \operatorname{var}\{m\}=\boldsymbol{E} m^{2}-(\boldsymbol{E} m)^{2}=\frac{\boldsymbol{E} s^{2}}{N} \Rightarrow \boldsymbol{E} m^{2}=(\boldsymbol{E} m)^{2}+\frac{\boldsymbol{E} s^{2}}{N}=\left(\mu+\frac{1}{2}\right)^{2}+\frac{\boldsymbol{E} s^{2}}{N} .
\end{aligned}
$$

Substitution of (14) in (13) results in

$$
\frac{N-1}{N} \boldsymbol{E} s^{2}+\frac{1}{N} \boldsymbol{E} s^{2}=\sigma^{2}+\mu^{2}+\mu+\frac{1}{6}-\mu^{2}-\mu-\frac{1}{4},
$$

or

$$
\boldsymbol{E} s^{2}=\sigma^{2}-\frac{1}{12} .
$$

Apparently $s^{2}$ is a negatively biased estimator of the population variance $\sigma^{2}$ in the above model, but this bias can be removed easily by replacing $s^{2}$ with $s^{2}+1 / 12$.

In this way the original bias is removed by the introduction of the variance component accounting for the within category variability of all ten intervals.

\section{References}

Cummins, R. A. (1995). On the trail of the gold standard for subjective well-being. Social Indicators Research, 35(2), 179-200. doi:10.1007/BF01079026.

Kalmijn, W. M. (2010). Quantification of happiness inequality, Ch. 6. PhD dissertation, Erasmus University Rotterdam, NL. ISBN 978-90-9025604-7. Available at http://repub.eur.nl/resource/pub_21777/index.html.

Kalmijn, W. M., Arends, L. R., \& Veenhoven, R. (2011). Happiness scale interval study. Methodological considerations. Social Indicators Research, 102(3), 497-515. doi:10.1007/s11205-010-9688-2.

Mazaheri, M., \& Theuns, P. (2009). Effects of varying response formats on self-ratings of life satisfaction. Social Indicators Research, 90, 381-395. doi:10.1007/s11205-008-9263-2. ISSN (on line) 1573-0921. 
Veenhoven, R. (1993). Happiness in nations: Subjective appreciation of life in 56 nations 1946-1992. RISBO, Studies in Sociale en Culturele Verandering, nr. 2, Erasmus University Rotterdam, NL. ISBN 90-72597-46.

Veenhoven, R. (2009). International scale interval study: Improving the comparability of responses to survey questions about happiness. In: Valerie Moller \& Dennis Huschka (Eds.) Quality of life and the millennium challenge: Advances in quality-of-life studies, theory and research, Social Indicators Research Series, vol. 35. Springer. e-ISBN 978-1-4020-8569-7.

Veenhoven, R. (2011). World database of happiness, continuous register of research on subjective appreciation of life. Erasmus University Rotterdam, NL. Available at http://www.worlddatabaseof happiness.eur.nl. 